\title{
Behaviour of Lagrangian triangular mixed fluid finite elements
}

\author{
S GOPALAKRISHNAN* and G DEVI ${ }^{\dagger}$ \\ Department of Aerospace Engineering, Indian Institute of Science, Bangalore \\ 560012 , India \\ $\dagger$ Present address: CADS Software Inc., Chennai 600 033, India \\ e-mail: krishnan@aero.iisc.ernet.in
}

MS received 26 November 1999; revised 6 December 1999

\begin{abstract}
The behaviour of mixed fluid finite elements, formulated based on the Lagrangian frame of reference, is investigated to understand the effects of locking due to incompressibility and irrotational constraints. For this purpose, both linear and quadratic mixed triangular fluid elements are formulated. It is found that there exists a close relationship between the penalty finite element approach that uses reduced/selective numerical integration to alleviate locking, and the mixed finite element approach. That is, performing reduced/selective integration in the penalty approach amounts to reducing the order of pressure interpolation in the mixed finite element approach for obtaining similar results. A number of numerical experiments are performed to determine the optimum degree of interpolation of both the mean pressure and the rotational pressure in order that the twin constraints are satisfied exactly. For this purpose, the benchmark solution of the rigid rectangular tank is used. It is found that, irrespective of the degree of mean and the rotational pressure interpolation, the linear triangle mesh, with or without central bubble function (incompatible mode), locks when both the constraints are enforced simultaneously. However, for quadratic triangle, linear interpolation of the mean pressure and constant rotational pressure ensures exact satisfaction of the constraints and the mesh does not lock. Based on the results obtained from the numerical experiments, a number of important conclusions are arrived at.
\end{abstract}

Keywords. Mixed finite elements; penalty approach; field consistency; reduced/selective integration; incompressibility constraint; irrotational constraint; fluid-structure interaction.

* For correspondence 


\section{Introduction}

Fluid finite elements are normally required to analyse problems involving dynamic interaction between flexible structures and the surrounding fluid medium, that is, the fluidstructure interaction problems. Most of the current fluid-structure analysis is based on the Eulerian-Lagrangian or the $u-p$ approach. In this approach, fluids are modelled based on the Eulerian frame of reference. As a result, pressures (or velocity potentials) become the nodal variables. The structures as usual are modelled based on the Lagrangian frame of reference and hence have displacements as nodal degrees of freedom. At the fluid-solid interface, the coupling is enforced by matching the normal velocities in fluid and solid domains. This results in matrices being unsymmetric and having large bandwidth, and hence special type of solvers are required to solve the coupled systems at the cost of extra computational effort. Hence, the method cannot be readily incorporated into any existing finite element software. The reader can refer to Zienkiewicz \& Taylor (1991) for more details on the approach.

Alternatively, both the fluid and the structure can be modelled with the Lagrangian frame of reference. This results in having displacements as degrees of freedom for both fluid and solid domains. Since the variables are identical in both the domains, no special coupling schemes are required and the compatibility and the equilibrium conditions are automatically satisfied at the fluid-solid interface through a matrix assembly procedure. These fluid elements, formulated based on the Lagrangian frame of reference, are also called "Mock Fluid Elements". They can be readily incorporated into any existing finite element software without much modification. Details of the approach are given in Cook et al (1989).

One of the problems associated with Lagrangian fluid elements is the presence of mesh locking due to incompressibility constraints (Wilson \& Khalvati 1983). That is, in the limit as the fluid becomes incompressible (Poisson's ratio $\nu \rightarrow 0.5$ ), the bulk modulus becomes infinite. This leads to zero volumetric strain. Hence, the fluid is constrained to exhibit zero volume change in the penalty limit. This causes the mesh to lock, giving displacements (velocities) that are several orders of magnitude less than their true values. Such behaviour is also seen in displacement-based structural elements for incompressible problems (Pian \& Lee 1976; Satish Chandra \& Prathap 1989).

Lagrangian fluid elements also have a tendency to exhibit zero energy modes and spurious acoustic or pressure modes. While the presence of the zero energy modes are inherent to the Lagrangian fluid element formulation due to circulation of the fluids, the spurious pressure modes are essentially due to higher order integration of the stiffness matrix (Wilson \& Khalvati 1983). Therefore, in order to eliminate the unwanted zero energy modes and identify the true pressure modes, Hamdi et al (1978) introduced rotational constraints, that is, assumed the fluid irrotational. Enforcing fluid irrotationality would mean introduction of additional constraints to an already volumetrically constrained fluid. Hence, Lagrangian fluid finite elements can be considered a constrained media problem having two naturally occurring constraints - the incompressibility constraints and the irrotational constraints.

The common methods available to alleviate locking are the penalty finite element approach, the field consistent approach and the mixed finite element approach. In the penalty approach, the stiffness matrix of a constrained system can be split into two parts the first due to constrained strain field and the second due to unconstrained strain field. The characteristic feature of a constrained system is that it gives rise to a part of the stiffness 
matrix, whose entries are very large compared to the other parts of the stiffness matrix derived from the unconstrained strain field. We call this matrix the penalty matrix. Such a system results in mesh locking unless the penalty matrix is singular. This singularity can be attained if the rank of the penalty matrix is lower than the order of the matrix, which can be ensured if the penalty matrix is under integrated. Hughes et al (1977) have shown that by using one-point integration of the shear energy terms of a linear shear flexible beam element, shear locking can be removed and the element can give superior performance.

However, fluid elements formulated based on Lagrangian frame of reference, has to not only satisfy the incompressibility constraints, but also the irrotational constraints simultaneously. In the discretized sense, it requires that the matrix obtained by summing of the volumetric stiffness matrix and the rotational stiffness matrix is singular. Previous papers by the authors (Gopalakrishnan \& Devi 1999) have shown that for fully integrated volumetric and rotational stiffness matrices for both linear and quadratic fluid triangular elements, the above condition is too difficult to achieve. This behaviour is attributed to the insufficient degrees of freedom available in these elements to satisfy the twin constraints. Note that the number of constraints an element has to satisfy is proportional to the number of integration points used to numerically evaluate the stiffness matrix. However, in the case of quadratic triangular fluid elements, it was shown that by performing selective integration (full 3-point integration on the volumetric stiffness matrix and reduced onepoint integration on the rotational stiffness matrix), the twin constraints were satisfied simultaneously and the element gave superior performance. Hence, it was shown that in cases where there are two or more naturally occurring constraints, locking has to be viewed in the global sense and hence constraint ratio (ratio of the number of active degrees of freedom in the model to the total number of constraints in the model) used as a measure to determine the presence of locking in the system. According to Cook et al (1989), the constraint ratio should always be greater than one and preferably two for two-dimensional elements.

The field consistency paradigm, propounded by Prathap (1993), requires that the interpolation function chosen to initiate the discretization process must also ensure that any special constraints that are anticipated be allowed for in a consistent way. Failure to do so causes solutions to lock. That is, the origin of the locking is linked to the interpolating functions of field displacement variables. If the interpolation function for the field variables contains some terms in excess (or missing) of that dictated by the consistency paradigm of the constrained strain field, the element will lock. Using this approach, Prathap \& Bhashyam (1982) were able to isolate those constants associated with the linear displacement field of a fully integrated Timoshenko beam element that caused locking. They also showed that the stiffness terms of the same element that introduce locking in the fully integrated case are not sensed when reduced integrated, thus giving superior performance. In the discretized sense, ignoring certain constants in the field variable(s) that are associated with the locking yields a penalty matrix that is rank deficient (or singular). This process enables satisfactory enforcement of the constraint. In other words, the consistency paradigm gives a more rational explanation on the success of reduced/selective integration procedures for constraint media problems. Prathap (1993), using the same approach, has explained the success of using incompatible modes (bubble function) in certain locking situations. In addition, it is also possible to quantify these errors associated with locking (Prathap 1999). Some of the elements formulated based on the consistency paradigm are given by Prathap \& Ramesh Babu (1986a) for higher order shear flexible beams, Prathap \& Ramesh Babu (1986b) for 
thick curved beams with shear and membrane locking, and Satish Chandra \& Prathap (1989) for locking in 3-D solids due to incompressibility.

One of the drawbacks of the penalty approach to fluids is that the mean pressure is not characterized properly. As a result, the pressure estimates, which are obtained through the differentiation of displacement field, are highly in error especially when the fluid is nearly incompressible. Hence, it is necessary that, for accurate solutions, not only the constraints be satisfied, but also the mean pressures be predicted accurately. This can be accomplished by the mixed finite element approach.

In the mixed finite element approach, the constraints are enforced by Lagrange multipliers. These multipliers, which have physical significance, are related to actual physical quantities appearing in the formulation of the problem. It can be seen later that for the case of fluids, the multiplier turns out to be pressure, which we try to characterize accurately. Hence, this method, due to introduction of Lagrange multipliers, increases the overall system size.

In this approach the original virtual work statement is augmented by the constraint equations, multiplied by Lagrange parameters. Minimization is done not only with respect to the primary displacement variables, but also the Lagrange multipliers. This process yields a variational statement followed by a set of constraint equations involving the multipliers. The number of constraint equations is equal to the number of Lagrange multipliers used (or number of constraints enforced). This variational procedure of enforcing constraints through Lagrange multipliers constitutes the $\mathrm{Hu}-\mathrm{Washizu}$ principle (Hu 1955). The main aim of this paper is to formulate both linear and quadratic mixed triangular fluid elements based on the $\mathrm{Hu}-$ Washizu principle and closely study its relationship with the penalty finite element approach. Since two constraints are required to be enforced simultaneously, three-field mixed elements involving the displacement and the two Lagrange multipliers, are formulated. The study also includes the effect of bubble functions (or incompatible modes) on the behaviour of the mixed elements. Based on the various numerical experiments conducted on these elements, a number of conclusions are drawn.

\section{Hu-Washizu variational statement}

The strain energy functional for a vibrating fluid is augmented by two constraint equations through two Lagrange multipliers $\lambda_{1}$ and $\lambda_{2}$ as follows:

$$
\begin{aligned}
\pi= & \frac{1}{2} \int_{V} \varepsilon_{v}^{T} p \mathrm{~d} V+\frac{1}{2} \int_{V} \varepsilon_{z}^{T} p_{z} \mathrm{~d} V+\frac{1}{2} \int_{V} \gamma_{x y}^{T} \tau_{x y} \mathrm{~d} V-\int_{V}\{u\}^{T}\{b\} \mathrm{d} V \\
& -\int_{S}\{u\}^{T}\{t\} \mathrm{d} S+\int_{V} \lambda_{1}\left(\varepsilon_{v}-\frac{p}{K}\right) \mathrm{d} V+\int_{V} \lambda_{2}\left(\varepsilon_{z}-\frac{p_{z}}{R}\right) \mathrm{d} V
\end{aligned}
$$

where

$$
\varepsilon_{v}=\frac{\partial u}{\partial x}+\frac{\partial v}{\partial y}, \quad \varepsilon_{z}=\frac{1}{2}\left[\frac{\partial u}{\partial y}-\frac{\partial v}{\partial x}\right], \quad \gamma_{x y}=\frac{\partial u}{\partial y}+\frac{\partial v}{\partial x} .
$$

Here $p, p_{z}$ and $\tau_{x y}$ are the mean pressure, rotational pressure and shear stress, $u$ and $v$ are the displacement components in the $x$ and $y$ directions and $\{u\}$ is the nodal displacement vector, with $\{b\}$ and $\{t\}$ being the body force and surface traction vectors respectively. The 
above functional is similar to the famous $\mathrm{Hu}-$ Washizu variational statement for fluids satisfying twin constraints.

If we look at the functional we see that there are three independent variables, namely $\{u\}, p$ and $p_{z}$. Minimizing with respect to these three variables we get

$$
\begin{aligned}
\int_{V} \delta \varepsilon_{v}^{T} p \mathrm{~d} V & +\int_{V} \delta \varepsilon_{z}^{T} p_{z} \mathrm{~d} V+\int_{V} \delta \gamma_{x y}^{T} \tau_{x y} \mathrm{~d} V-\int_{V}\{\delta u\}^{T}\{b\} \mathrm{d} V-\int_{S}\{\delta u\}^{T}\{t\} \mathrm{d} S \\
& +\int_{V} \lambda_{1} \delta \varepsilon_{v} \mathrm{~d} V+\int_{V} \lambda_{2} \delta \varepsilon_{z} \mathrm{~d} V=0 \\
& \int_{V} \varepsilon_{v}^{T} \delta p \mathrm{~d} V+\int_{V} \lambda_{1}\left(-\frac{\delta p}{K}\right) \mathrm{d} V=0 \\
& \int_{V} \varepsilon_{z}^{T} \delta p_{z} \mathrm{~d} V+\int_{V} \lambda_{2}\left(-\frac{\delta p_{z}}{R}\right) \mathrm{d} V=0
\end{aligned}
$$

From the last two conditions we get $\lambda_{1}=p$, the mean pressure and $\lambda_{2}=p_{z}$ the rotational pressure. It is to be noted that these conditions can now be written as

$$
\begin{aligned}
& \int_{V} \delta p\left(\varepsilon-\frac{p}{K}\right) \mathrm{d} V, \quad \text { and } \\
& \int_{V} \delta p_{z}\left(\varepsilon_{z}-\frac{p_{z}}{R}\right) \mathrm{d} V .
\end{aligned}
$$

The slosh energy and kinetic energy functionals are given by

$$
\pi_{S}=\frac{1}{2} \int_{S} \varrho g\left\{u_{S}\right\}^{T}\left\{u_{S}\right\} \mathrm{d} S, \quad T=\frac{1}{2} \int_{V} \varrho\{V\}^{T}\{V\} \mathrm{d} V,
$$

where $\left\{u_{s}\right\}$ is the surface displacement vector on the surface $S, \varrho$ is the density of the fluid, $g$ is the acceleration due to gravity and $\{V\}=\{\dot{u} \dot{v}\}$ is the velocity vector.

\section{Mixed finite element formulation}

Both 3-noded linear triangular and 6-noded isoparametric quadratic triangular fluid elements are formulated. Each node has two degrees of freedom and the displacement field in each of these can be expressed in matrix form as

$$
\left\{\begin{array}{l}
u \\
v
\end{array}\right\}=\left[\begin{array}{ll}
{[N]^{T}} & {[0]^{T}} \\
{[0]^{T}} & {[N]^{T}}
\end{array}\right]\{d\},
$$

where $u(x, y)$ and $v(x, y)$ are the displacement fields in $x$ and $y$ directions, $\{d\}$ is nodal displacement vector and $[N]$ is shape function matrix. For the linear triangle, it is given by

$$
[N]^{T}=\left[L_{1} L_{2} L_{3}\right]
$$

For the quadratic triangle, the shape function matrix is given by

$$
[N]^{T}=\left[\begin{array}{lll}
L_{1}\left(2 L_{1}-1\right) & 4 L_{1} L_{2} \cdots L_{3}\left(2 L_{3}-1\right) & 4 L_{3} L_{1}
\end{array}\right] .
$$

Here, $L_{i}$ is the area coordinate. In this approach, in addition to the displacements, both mean pressure and rotational pressure need to be interpolated. Pressure variation can be 
symbolically written as

$$
p(x, y)=\left[N_{P}\right]^{T}\{p\}, \quad p_{z}(x, y)=\left[N_{p z}\right]^{T}\left\{p_{z}\right\},
$$

where $\left[N_{p}\right]$ and $\left[N_{p z}\right]$ are shape functions for mean and rotational pressures and $\{p\}$ and $\left\{p_{z}\right\}$ are nodal mean and rotational pressures respectively. In this study, constant, linear and quadratic pressure interpolations are considered. The shape function matrices for linear and quadratic variation are similar to the displacement shape function matrices given in (9) and (10) respectively. For constant pressure interpolation, the shape function matrices take the form

$$
\left[N_{p}\right]^{T}=\left[N_{p z}\right]^{T}=[1] .
$$

Using the displacement field given by (8), the volumetric, rotational and shear strains become

$$
\begin{aligned}
\varepsilon_{v} & =\frac{\partial u}{\partial x}+\frac{\partial v}{\partial y}=\left[B_{v}\right]\{u\}, \quad \varepsilon_{z}=\frac{1}{2}\left[\frac{\partial u}{\partial y}-\frac{\partial v}{\partial x}\right]=\left[B_{z}\right]\{u\}, \\
\gamma_{x y} & =\frac{\partial u}{\partial y}+\frac{\partial v}{\partial x}=\left[B_{x y}\right]\{u\} .
\end{aligned}
$$

Substituting the above strain fields and the discretized pressures in the $\mathrm{Hu}$-Washizu variational statement, we get the following matrix equilibrium equations

$$
\left[\begin{array}{ccc}
{[M]} & {[0]} & {[0]} \\
{[0]} & {[0]} & {[0]} \\
{[0]} & {[0]} & {[0]}
\end{array}\right]\left\{\begin{array}{c}
\{\ddot{u}\} \\
\{p\} \\
\{p z\}
\end{array}\right\}+\left[\begin{array}{ccc}
{\left[K_{u u}\right]} & {\left[K_{u p}\right]} & {\left[K_{u p z}\right]} \\
{\left[K_{u p}\right]^{T}} & {\left[K_{p p}\right]} & {[0]} \\
{\left[K_{u p z}\right]^{T}} & {[0]} & {\left[K_{p z p z}\right]}
\end{array}\right]\left\{\begin{array}{c}
\{u\} \\
\{p\} \\
\{p z\}
\end{array}\right\}=\left\{\begin{array}{l}
\{f\}_{1} \\
\{f\}_{2} \\
\{f\}_{3}
\end{array}\right\}
$$

where

$$
\begin{aligned}
{[M] } & =\int_{V} \rho[N]^{T}[N] \mathrm{d} V \\
{\left[K_{u u}\right] } & =\int_{V} G\left[B_{x y}\right]^{T}\left[B_{x y}\right] \mathrm{d} V, \quad\left[K_{u p}\right]=\int_{V}\left[B_{v}\right]^{T}\left[N_{p}\right] \mathrm{d} V, \quad\left[K_{p p}\right]=\int_{V} \frac{1}{K}\left[N_{p}\right]^{T}\left[N_{p}\right] \mathrm{d} V \\
{\left[K_{u p z}\right] } & =\int_{V}\left[B_{z}\right]^{T}\left[N_{p z}\right] \mathrm{d} V, \quad\left[K_{p z p z}\right]=\int_{V} \frac{1}{R}\left[N_{p z}\right]^{T}\left[N_{p z}\right] \mathrm{d} V \\
\{f\}_{1} & =\int_{V}[N]\{u\}^{T}\{b\} \mathrm{d} V+\int_{S}\{N\}\{u\}^{T}\{t\} \mathrm{d} S, \quad\{f\}_{2}=0 \text { and }\{f\}_{3}=0
\end{aligned}
$$

Here $G$ is the shear modulus, $\rho$ is the density of the fluid, $K$ is the bulk modulus and $R=\alpha K$ is the rotational modulus. The value of $\alpha$ is taken as 100 as suggested by Wilson \& Khalvati (1983). In addition to the above, the slosh matrix, obtained by the minimization of the slosh energy functional, is given by

$$
\left[K_{S}\right]=\int_{S} \rho g\left[N_{S}\right]^{T}\left[N_{S}\right] \mathrm{d} S,
$$

and added to the surface terms of the element stiffness matrix. $\left[N_{S}\right]$ is the shape function matrix used for the interpolation of surface displacement. For linear triangles, the fluid 
surface is modelled as a two-noded line element with only vertical degrees of freedom. The shape function for this surface element is given by

$$
\left[N_{S}\right]^{T}=\left[\left(1-\frac{x}{b}\right) \frac{x}{b}\right] .
$$

Here, $x$ is the axial coordinate, $b$ is the element length, and $g$ is the acceleration due to gravity. Using (18) in (17), the slosh stiffness matrix for the linear triangle becomes

$$
\left[K_{S}\right]=\frac{\rho g b}{6}\left[\begin{array}{ll}
2 & 1 \\
1 & 2
\end{array}\right] .
$$

In the case of quadratic triangular elements, the fluid surface is modelled as a 3-noded quadratic line element, whose shape function is given by

$$
\left[N_{S}\right]^{T}=\left[\begin{array}{lll}
\xi\left(\frac{\xi-1}{2}\right) & 1-\xi^{2} & \xi\left(\frac{\xi+1}{2}\right)
\end{array}\right]^{T}, \quad \xi=(2 x / b-1) .
$$

The slosh stiffness matrix in this case becomes

$$
\left[K_{S}\right]=\frac{\rho g b}{30}\left[\begin{array}{ccc}
4 & 2 & -1 \\
2 & 16 & 1 \\
-1 & 1 & 4
\end{array}\right] .
$$

The above equations give rise to many exciting possibilities. Since the pressures are also unknown, we have the option of maintaining continuity of pressures or condensing them out before assembly. In addition, pressure interpolation could be of any order independent of displacement interpolation. However, there are sets of rules while choosing the interpolation order for the secondary variables. In this study, the pressure continuity is not maintained at the nodes and these variables are condensed out at the element level before assembly.

The key to the success of the mixed finite element approach is to choose the appropriate interpolation for the displacements and pressures. From the literature (Bathe 1997), it is seen that the choice of the appropriate pressure interpolation is not obvious and is indeed much more difficult. A lower order pressure interpolation leads to spurious pressure or acoustic modes. On the other hand, pressures should also not be interpolated at too high a degree because the element then behaves like displacement-based elements and locks. Hence the highest degree of pressure interpolation that does not introduce locking into the element needs to be used. The general condition to be satisfied about the number of degrees of freedom is $n_{u} \geq n_{p},\left[K_{u p}\right]\{p\} \neq 0$ for all $p \neq 0$ and $\left[K_{u p z}\right]\left\{p_{z}\right\} \neq 0$ for all $p_{z} \neq 0$ to prevent instability. Here $n_{u}$ is the number of displacement degrees of freedom, $n_{p}$ is the number of pressure (mean) degrees of freedom and $n_{p z}$ is the number of rotational pressure degrees of freedom.

\section{Numerical studies and discussion}

The behaviour of both the elements (linear and quadratic) is studied for different combinations of mean and rotational pressure interpolations. For some cases in the penalty approach, incompatible modes are introduced to alleviate locking. In the global sense, this procedure increases the constraint ratio. In this study, the effects of incompatible modes on the performance of the mixed elements will also be investigated for various mean and 


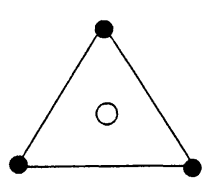

Linear triangle with constant pressure

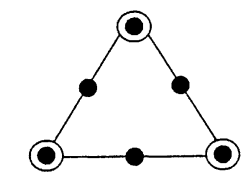

Quadratic triangle with linear pressure

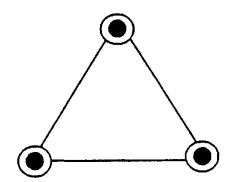

Linear triangle with linear pressure

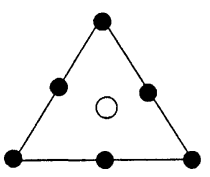

Quadratic triangle with constant pressure

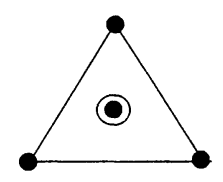

Linear triangle with one bubble function and constant pressure
- Displacement node

Pressure nodes

Figure 1. Element configurations for numerical studies.

rotational pressure interpolations. Some of the element configurations studied here are schematically shown in figure 1 . All the matrices in (14)-(16) are numerically integrated. The order of integration of various matrices is given in table 1. All the numerical studies in this paper are performed on a rigid rectangular tank problem, the tank being full of water. The exact slosh and acoustic frequencies for this problem are given in Abramson (1966) and Olson \& Bathe (1983) respectively. These are given by

$$
\begin{gathered}
\omega_{\text {slosh }}^{2}=g k \tanh (k h), \quad k^{2}=\pi^{2}\left(\left(\frac{m}{b}\right)^{2}+\left(\frac{n}{h}\right)^{2}\right), \quad m=n=0,1,2, \ldots, \\
\lambda=\omega^{2}=\frac{K}{\rho} \pi^{2}\left(\frac{m^{2}}{b^{2}}+\frac{n^{2}}{4 h^{2}}\right), \quad m=n=0,1,2 \ldots
\end{gathered}
$$

The following properties of the tank were used for all the examples.

Tank width $b=5.080 \mathrm{~m}$; tank depth $h=1.905 \mathrm{~m}$;

density $\rho=1000 \mathrm{~kg} / \mathrm{m}^{3}$; bulk modulus $K=207 \mathrm{GPa}$;

acceleration due to gravity $g=9.81 \mathrm{~m} / \mathrm{s}^{2}$.

\subsection{Linear triangle without bubble function}

Constraint equations (5) and (6) dictate the exact order of mean and rotational pressure interpolations, required for convergence of the solutions. The volumetric strain $\varepsilon_{V}$ and the

\begin{tabular}{|c|c|c|c|c|c|c|c|}
\hline \multirow[b]{3}{*}{ Matrices } & \multicolumn{7}{|c|}{ Pressure variation } \\
\hline & \multicolumn{2}{|c|}{ Linear triangle } & \multicolumn{3}{|c|}{ Linear triangle +1 bubble function } & \multicolumn{2}{|c|}{ Quadratic triangle } \\
\hline & Constant & Linear & Constant & Linear & Quadratic & Constant & Linear \\
\hline$\left[\mathbf{K}_{u p}\right]$ & 1 & 1 & 3 & 4 & 6 & 1 & 3 \\
\hline$\left[\mathbf{K}_{p p}\right]$ & 1 & 3 & 1 & 3 & 6 & 1 & 3 \\
\hline$\left[\mathbf{K}_{u u}\right]$ & 1 & 1 & 6 & 6 & 6 & 3 & 3 \\
\hline$[\mathbf{M}]$ & 3 & 3 & 12 & 12 & 12 & 6 & 6 \\
\hline
\end{tabular}

Table 1. Order of integration for various matrices and pressure interpolations. 


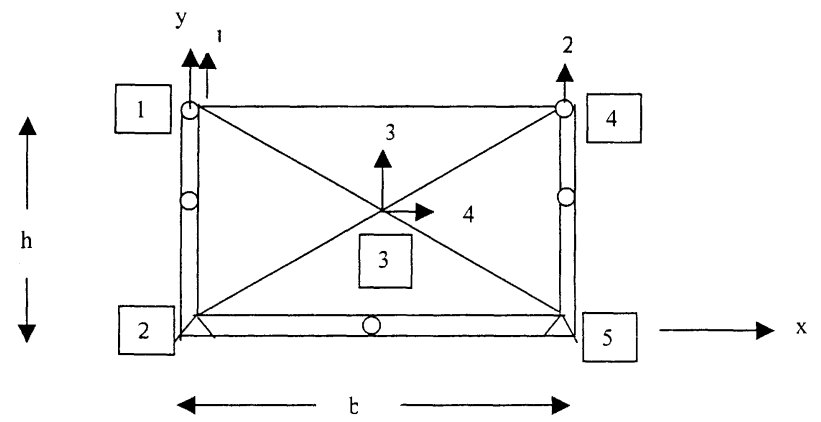

Figure 2. Rectangular rigid tank modelled by four elements.

rotational strain $\varepsilon_{z}$ are derived from the displacement field, and the pressure fields $p$ and $p_{z}$ are interpolated separately. According to Prathap (1999), constraint equations (5) and (6) represent orthogonality conditions when the pressure variation is represented by some orthogonal functions. For rectangular elements, $p$ (or $p_{z}$ ) can be represented by a set of Legendre polynomials. When such a variation of the secondary variables is substituted in the constraint equations, the variation of the displacement field, consistent with the constraint imposed, is obtained.

In the triangular domain, the above constraint equations can effectively fix the pressure interpolation required to get good results. For linear triangles without any bubble functions,

$$
\varepsilon_{v}=\frac{\partial u}{\partial x}+\frac{\partial v}{\partial y}=\text { constant }
$$

Hence, constant pressure is the ideal choice to match the constant $\varepsilon_{v}$ obtained from displacement interpolation. By similar argument, constant rotational pressure is required for balancing constant rotational strain. Any interpolations of pressure greater than the required degree may lead to locking.

In order to study the difference in behaviour between the penalty finite element approach (Gopalakrishnan \& Devi 1999a) and the formulated mixed linear element, a rigid rectangular tank modelled by four elements (figure 2) is considered. The boundary conditions are such that the fluid can slip along both the vertical as well as the horizontal faces of the tank. Hence, there are only four active degrees of freedom in the finite element model. Here, both the constant and linear variation of the pressures is considered. All the

Table 2. Comparison of frequencies for linear triangle.

\begin{tabular}{|c|c|c|c|c|c|c|c|}
\hline \multirow{2}{*}{\multicolumn{2}{|c|}{$\begin{array}{l}\text { Penalty approach } \\
\text { frequencies }(\mathrm{rad} / \mathrm{s})\end{array}$}} & \multicolumn{6}{|c|}{ Mixed approach frequencies ( $\mathrm{rad} / \mathrm{s})$} \\
\hline & & \multicolumn{2}{|c|}{ Constant $p$, constant $p_{z}$} & \multicolumn{2}{|c|}{ Linear $p$, linear $p_{z}$} & \multirow{2}{*}{$\begin{array}{l}\text { Constant } p, \\
\text { linear } p_{z} \\
R=100 \mathrm{~K}\end{array}$} & \multirow{2}{*}{$\begin{array}{c}\begin{array}{c}\text { Linear } p \\
\text { constant } p_{z}\end{array} \\
R=100 K\end{array}$} \\
\hline$R=0$ & $R=100 K$ & $R=0$ & $R=100 K$ & $R=0$ & $R=100 K$ & & \\
\hline 2.0190 & 1289.6 & 2.0190 & 1289.6 & 2.0190 & 1289.6 & 1289.6 & 1289.6 \\
\hline 1224.6 & 4256.6 & 1224.6 & 4256.6 & 1224.6 & 4256.6 & 4256.6 & 4256.6 \\
\hline 1800.7 & 7971.3 & 1800.7 & 7971.3 & 1800.7 & 7971.3 & 7671.3 & 7971.3 \\
\hline 3950.1 & 13424 & 3950.1 & 13423 & 3950.1 & 13423 & 13424 & 13423 \\
\hline
\end{tabular}


Table 3. Comparison of frequencies for linear triangle with one bubble function

\begin{tabular}{|c|c|c|c|c|c|}
\hline \multirow{2}{*}{\multicolumn{2}{|c|}{$\begin{array}{l}\text { Penalty approach } \\
\text { frequencies }(\mathrm{rad} / \mathrm{s})\end{array}$}} & \multicolumn{4}{|c|}{ Mixed approach frequencies (rad/s) } \\
\hline & & \multicolumn{2}{|c|}{ Quadratic $p$, quadratic $p_{z}$} & \multirow{2}{*}{$\begin{array}{l}\text { Quadratic } p, \\
\text { linear } p_{z} \\
R=100 \mathrm{~K}\end{array}$} & \multirow{2}{*}{$\begin{array}{c}\begin{array}{c}\text { Linear } p \\
\text { quadratic } p_{z}\end{array} \\
R=100 \mathrm{~K}\end{array}$} \\
\hline$R=0$ & $R=100 K$ & $R=0$ & $R=100 K$ & & \\
\hline 2.0190 & 1289.6 & 2.0155 & 1268.2 & 1268.2 & 1289.6 \\
\hline 1224.6 & 4256.6 & 1202.1 & 4226.0 & 4226.0 & 4256.6 \\
\hline 1800.7 & 7971.3 & 1790.3 & 8314.9 & 8314.9 & 7971.3 \\
\hline 3950.1 & 13424 & 4126.5 & 13380 & 13380 & 13424 \\
\hline
\end{tabular}

matrices appearing in (15) are numerically integrated using the one-point integration scheme for both the cases.

Table 2 gives the comparison of results obtained from the penalty approach and the present approach. The exact value of the fundamental slosh and acoustic modes are $2.24 \mathrm{rad} / \mathrm{s}$ and $1186 \mathrm{rad} / \mathrm{s}$, respectively. From table 2 , it is clear that for $R=0$ and $R=100 \mathrm{~K}$ the results for all the cases are exactly similar to the penalty approach results. That is, higher pressure interpolation has no effect and the presence of twin constraints locks the mesh. It is also found that for the case with $R=0$, the slosh frequency has about $10 \%$ error compared to $3 \%$ error obtained for fundamental acoustic frequency. This is expected because, in a linear triangle, there are fewer degrees of freedom and the displacement interpolation is of very low order.

\subsection{Linear triangle with one central bubble function}

In this case, one cubic bubble of shape function $27 L_{1} L_{2} L_{3}$ is added to increase the degree of displacement interpolation. This would require both the pressure variations to be quadratic. Here, a number of possibilities exist as before. As in the previous case, a rigid rectangular tank is modelled by four mixed linear triangular elements (figure 2). The integration orders for various matrices are given in table 1. Table 3 gives the comparisons of the frequencies obtained for few critical pressure interpolations. The bubble functions here are dynamically condensed out before assembly. From the table it is found that the element locks when twin constraints are enforced simultaneously. That is, the introduction of bubble function does not alleviate locking. In addition, only linear mean pressure variation is able to give the solutions predicted by the penalty approach. The point worthy of note is that this happens even when the rotational pressure is interpolated quadratically. Additionally, it is found that the results predicted by the quadratic mean and rotational pressure variation and the quadratic mean pressure and linear rotational pressure variation produce exactly the same results, which are different from the penalty approach results. Hence, the accuracy of the result mainly hinges on how well the mean pressure is interpolated. Errors in the frequencies obtained are of similar order as obtained in the linear triangle case without any bubble function.

\subsection{Quadratic triangular element}

A quadratic triangular element based on penalty approach was formulated by Gopalakrishnan \& Devi (1999b). They showed that in presence of the twin constraints 
of incompressibility and irrotationality, the element behaved very well when selective integration procedure (full 3-point integration on the volumetric stiffness and reduced 1-point integration on the rotational stiffness) was performed. In the global sense, this scheme gave high constraint ratio. In the present context, it would be interesting to establish the relationship between the penalty approach using selective integration, reduced integration etc. and the mixed approach with appropriate pressure interpolation (both $p$ and $p_{z}$ ).

By looking at the constraint equations, (5) and (6), it is obvious that the appropriate order of mean pressure and rotational pressure is linear. The objective of the whole exercise is to obtain good estimates of slosh and acoustic modes when twin constraints are enforced simultaneously. In order to meet this objective, one has to perform studies on various pressure interpolations and determine what orders of mean and rotational pressures are required for satisfaction of both constraints. For this study the same four-element mesh shown in figure 2 is used. This mesh has 17 active degrees of freedom. The integration orders for various matrices are given in table 1. Table 4 gives the results for constant mean pressure and constant rotational pressure interpolation.

The answers are compared with those of the penalty approach with reduced integration of both volumetric and rotational stiffness terms. We find that the results of these two approaches are similar and the results are greatly in error due to the presence of spurious zero energy modes arising due to very low pressure interpolation. The curious thing is that most of the acoustic modes are not spurious.

Table 5 shows the comparison of results between penalty approach with full integration (both volumetric and rotational stiffness terms) and mixed approach with linear variation of both mean and rotational pressures. Note that the pressure variation considered in this case is same as what is dictated by the constraint equations (5) and (6), respectively. From the table it is clear that the penalty approach results are the same as the mixed approach with linear variations of pressure. With compressibility constraint alone, the mesh does not lock and slosh and acoustic mode estimates are quite good. When rotational constraint is enforced, all zero energy modes vanish and the mesh locks. However, only two acoustic modes (second and fourth) are identified as the true ones.

Table 4. Comparison of frequencies for quadratic triangle with constant mean and rotational pressures.

\begin{tabular}{|c|c|c|c|}
\hline \multicolumn{2}{|c|}{$\begin{array}{l}\text { Penalty approach frequencies (rad/s) } \\
\text { (reduced integration) }\end{array}$} & \multicolumn{2}{|c|}{$\begin{array}{l}\text { Mixed approach frequencies (rad/s) } \\
\quad\left(\text { constant } p \text {, constant } p_{z}\right)\end{array}$} \\
\hline$R=0$ & $R=100 K$ & $R=0$ & $R=100 K$ \\
\hline 11 zero-energy modes & 7 zero-energy modes & 11 zero-energy modes & 7 zero-energy modes \\
\hline 4.1868 & 4.1793 & 4.1869 & 4.1793 \\
\hline 7.1778 & 7.0833 & 7.1778 & 7.0833 \\
\hline 1779.3 & $1776.5^{*}$ & 1779.3 & $1776.5^{*}$ \\
\hline 3373.6 & $3373.4^{*}$ & 3373.6 & $3373.4^{*}$ \\
\hline 3535.4 & $3534.7^{*}$ & 3535.4 & $3534.7^{*}$ \\
\hline 7313.4 & $7265.4^{*}$ & 7313.4 & $7265.4^{*}$ \\
\hline - & 14778 & - & 14778 \\
\hline - & 20944 & - & 20944 \\
\hline- & 32791 & - & 32791 \\
\hline - & 39017 & - & 39017 \\
\hline
\end{tabular}

${ }^{*}$ True acoustic modes 
Table 5. Comparison of frequencies for quadratic triangle with linear mean and rotational pressures.

Penalty approach frequencies $(\mathrm{rad} / \mathrm{s})$ (full integration)

\begin{tabular}{cccc}
\hline$R=0$ & $R=100 K$ & $R=0$ & $R=100 K$ \\
\hline 4 zero-energy modes & 481.00 & 4 zero-energy modes & 481.0 \\
\cline { 2 - 3 } 2.2493 & $1188.5^{*}$ & 2.2493 & $1188.5^{*}$ \\
3.1809 & 1593.6 & 3.1809 & 1593.5 \\
1186.9 & 3920.4 & 1186.9 & 3920.4 \\
1487.1 & $4019.8^{*}$ & 1487.1 & $4019.8^{*}$ \\
2335.2 & 5622.1 & 2335.2 & 5622.1 \\
2460.9 & 8664.5 & 2460.9 & 8664.5 \\
3689.4 & 10141 & 3689.4 & 10141 \\
4004.9 & 12031 & 4004.9 & 12031 \\
4416.8 & 12750 & 4416.8 & 12750 \\
5055.1 & 16153 & 5055.1 & 16153 \\
6370.5 & 17907 & 6370.5 & 17907 \\
7332.6 & 19057 & 7332.6 & 19057 \\
9644.2 & 27656 & 9644.2 & 27656 \\
- & 30618 & - & 30618 \\
- & 42445 & - & 42445 \\
- & 46488 & - & 46488 \\
\hline
\end{tabular}

* True acoustic modes

From table 4 , it is clear that by use of constant $p$ and constant $p_{z}$ instead of the required linear variation, the results are similar to the reduced integrated penalty approach results. Again from table 5, the use of linear $p$ and linear $p_{z}$ variation is similar to using fully

Table 6. Comparison of frequencies for quadratic triangle with linear mean and constant rotational pressures.

\begin{tabular}{cccc}
\hline $\begin{array}{c}\text { Penalty approach frequencies }(\mathrm{rad} / \mathrm{s}) \\
\text { (selective integration } \mathrm{I})\end{array}$ & $\begin{array}{c}\text { Mixed approach frequencies }(\mathrm{rad} / \mathrm{s}) \\
\left.\text { (linear } p \text { constant } p_{z}\right)\end{array}$ \\
\hline$R=0$ & $R=100 \mathrm{~K}$ & $R=0$ & $R=100 \mathrm{~K}$ \\
\cline { 2 - 4 } 4 zero-energy modes & 2.2425 & 4 zero-energy modes & 2.2425 \\
2.2493 & 3.1809 & 2.2493 & 3.1809 \\
3.1809 & $1186.9^{*}$ & 3.1809 & $1186.9^{*}$ \\
1186.9 & $1479.2^{*}$ & 1186.9 & $1479.2^{*}$ \\
1487.1 & 2247.4 & 1487.1 & 2247.4 \\
2335.2 & $2335.1^{*}$ & 2335.2 & $2335.1^{*}$ \\
2460.9 & 2832.8 & 2460.9 & 2832.8 \\
3689.4 & $3688.0^{*}$ & 3689.4 & $3688.0^{*}$ \\
4004.9 & $4011.9^{*}$ & 4004.9 & $4011.9^{*}$ \\
4416.8 & $5041.5^{*}$ & 4416.8 & $5041.5^{*}$ \\
5055.1 & $6273.6^{*}$ & 5055.1 & $6273.6^{*}$ \\
6370.5 & $7290.3^{*}$ & 6370.5 & $7290.3^{*}$ \\
7332.6 & 9383.1 & 7332.6 & 9383.1 \\
9644.2 & 15167 & 9644.2 & 15167 \\
- & 21078 & - & 21078 \\
- & 32808 & - & 32808 \\
- & 39021 & - & 39021 \\
\hline
\end{tabular}

\footnotetext{
* True acoustic modes
} 
Table 7. Comparison of frequencies for quadratic triangle with constant mean and linear rotational pressures.

\begin{tabular}{|c|c|c|c|}
\hline \multicolumn{2}{|c|}{$\begin{array}{l}\text { Penalty approach frequencies }(\mathrm{rad} / \mathrm{s}) \\
\text { (selective integration II) }\end{array}$} & \multicolumn{2}{|c|}{$\begin{array}{l}\text { Mixed approach frequencies }(\mathrm{rad} / \mathrm{s}) \\
\left.\text { (constant } p \text {, linear } p_{z}\right)\end{array}$} \\
\hline$R=0$ & $R=100 K$ & $R=0$ & $R=100 K$ \\
\hline 11 zero-energy modes & 1 zero-energy mode & 11 zero-energy modes & 1 zero-energy mode \\
\hline 4.1868 & 1.6310 & 4.1869 & 1.6310 \\
\hline 7.1778 & 1023.2 & 7.1778 & 1023.2 \\
\hline 1779.3 & 2291.4 & 1779.3 & 2291.4 \\
\hline 3373.6 & 2293.1 & 3373.6 & 2293.1 \\
\hline 3535.4 & 5000.3 & 3535.4 & 5000.3 \\
\hline 7313.4 & 7940.9 & 7313.4 & 7940.9 \\
\hline- & 8831.1 & - & 8831.1 \\
\hline - & 10045.0 & - & 10045 \\
\hline - & 12750 & - & 12750 \\
\hline - & 16074 & - & 16074 \\
\hline- & 17365 & - & 17365 \\
\hline - & 17566 & - & 17566 \\
\hline - & 27627 & - & 27627 \\
\hline - & 30610 & - & 30610 \\
\hline - & 42383 & - & 42383 \\
\hline- & 46472 & - & 46472 \\
\hline
\end{tabular}

integrated volumetric and rotational stiffness as the penalty approach. Hence it is necessary to study the use of constant $p_{z}$ and linear $p$ variation, and constant $p$ and linear $p_{z}$ variation and see what these correspond to in terms of the penalty approach. Tables 6 and 7 give the results for these two cases.

From the above it is clear that, when linear $p$ and constant $p_{z}$ are used, the results correspond to the selective integration I result of the penalty approach (i.e. when volumetric stiffness is fully integrated (3-point integration) and rotational stiffness is reduced integrated (1-point integration)). This is expected and is in trend with the previous two cases. The slosh and acoustic mode estimates are very good. It is also found that there are very few spurious acoustic modes. Again from table 7, when the mean pressure interpolation is constant and rotational pressure is linear, as expected the results match the selective integration II (volumetric stiffness is reduced integrated and rotational stiffness is fully integrated) results. These results are not accurate. In addition, all the acoustic modes are spurious.

Hence, from this study it is clear that reducing the pressure interpolation in mixed formulation amounts to reducing the number of integration points in the penalty approach. The pressure interpolation dictated by the constraint equations yields a stiffness matrix that is similar to fully integrated volumetric and rotational stiffness in the penalty finite element approach. The above observation was by Zienkiewicz \& Taylor (1991b) when only one constraint was present.

\section{Concluding remarks}

The study on the mixed triangular elements has shown that there exists a close relationship between the mixed finite element approach and the penalty approach. It is found that 
reduced integration in the penalty approach means reduction in the pressure order of interpolation in the mixed finite element approach to get similar results. The degree of mean pressure interpolation is very critical for obtaining good frequency estimates. The study has shown that lower order pressure interpolation does not introduce spurious pressure modes. It is also found that the degree of rotational pressure interpolation is not so critical for obtaining good frequency estimates. For triangular elements, the minimum order of pressure interpolation is dictated by the constraint equations that emerge from the $\mathrm{Hu}-$ Washizu variational statement. That is, if the pressures (both mean and rotational) are interpolated as dictated by the constraint equations, it gives a stiffness matrix that corresponds to a fully integrated stiffness matrix of the penalty finite element approach. The study has shown that no pressure interpolation will help avoid locking in linear triangles, when the twin constraints are enforced simultaneously. However, in quadratic triangles, using linear interpolation for mean pressure and constant rotational pressure, locking in the element is removed under the action of twin constraints.

From this study, a concept has emerged that would unify the penalty finite approach, the mixed finite element approach and the field consistency approach. The field consistent method can be viewed both as penalty method and mixed finite element method. When viewed as a penalty method, the field consistency approach identifies constants in the displacement polynomial that are spurious and cause locking. It also suggests the required order of numerical integration in order to make the penalty matrix singular, which is the essential requirement for proper enforcement of constraints. In addition, through a functional re-constitution procedure (see Prathap 1993) one can also obtain the error measure that is associated with locking. The variational correctness of the field consistency is seen when one views it in mixed finite element context. That is, the constraint equations that emerge due to the minimization of secondary variables are, in fact, the variational representation of the field consistent approach. If one can identify the form of the constrained field involving arbitrary coefficients so that only the consistent terms are retained, then all the true constraints appear in the penalty limits. This form helps to determine the arbitrary coefficients in the redistributed field from the coefficients of the corresponding strain field derived directly from the kinematically admissible displacement fields using the constraint conditions. The integration of energies based on this form now yield a stiffness matrix, which is free of all inconsistencies. Both the known methods - the penalty method and the mixed element method - try to achieve this in one form or the other.

\section{References}

Abramson H N 1966 The dynamic behavior of liquids in moving containers. NASA SP-106

Bathe K J 1997 Finite element procedures (New Delhi: Prentice Hall of India)

Cook R D, Malkus D S, Plesha M E 1989 Concepts and application of finite element analysis (New York: John Wiley \& Sons)

Gopalakrishnan S, Devi G 1999a Constraints and mesh locking mechanism in Lagrangian triangular fluid finite elements. Int. J. Comput. Eng. Sci. (submitted)

Gopalakrishnan S, Devi G 1999b A Lagrangian quadratic triangular fluid finite element for fluidstructure interaction problems. Int. J. Comput. Eng. Sci. (submitted)

Hamdi M A, Ousset T, Verchery G 1978 A displacement method for the analysis of vibrations of coupled fluid-structure systems. Int. J. Numer. Methods Eng. 13: 139-150

Hu H C 1955 On some variational principles in the theory of elasticity and the theory of plasticity. Sci. Sin. 4: 33-54 
Hughes T G R, Taylor R L, Kanoknukulchal W 1977 A simple and efficient finite element for plate bending. Int. J. Numer. Methods Eng. 11: 1529-1543

Olson G L, Bathe K J 1983 A study of displacement based fluid finite elements for calculating frequencies of the fluid and fluid-structure systems. Nucl. Eng. Des. 76: 137-151

Pian T H H, Lee S W 1976 Notes on finite elements for nearly incompressible materials. AIAA J. 14: 824-826

Prathap G 1993 The finite element method in structural mechanics (Dordrecht: Kluwer Academic Press)

Prathap G 1999 A priori error estimation of finite element models from first principles. Sädhanā 24: 199-214

Prathap G, Bhashyam G R 1982 Reduced integration and the shear-flexible beam element. Int. J. Numer. Methods Eng. 18: 211-243

Prathap G, Ramesh Babu C 1986a Field consistent strain interpolation for quadratic shear flexible beam element. Int. J. Numer. Methods Eng. 23: 1973-1984

Prathap G, Ramesh Babu C 1986b An isoparametric quadratic thick curved beam element. Int. J. Numer. Methods Eng. 23: 1583-1600

Satish Chandra, Prathap G 1989 A field consistent formulation for the eight noded solid finite element. Comput. Struct. 33: 345-355

Wilson E L, Khalvati M 1983 Finite elements for dynamic analysis of fluid-solid systems. Int. J. Numer. Methods Eng. 19: 1657-1668

Zienkiewicz O C, Taylor R L 1991 The finite element method (New York: McGraw Hill) vol. 2 